jibbed at the very high price of S.B. they might well decide to get H.S. Those who want to have some values for heteromolecules without the need to use the polarizability coefficients, would choose C.S. C. A. Coulson (most important) with a comprehensive index. It should without doubt find a place in the library of any institute or scientist interested in reactor physics. Although it is not cheap at $£ 6$, it is very good value. M. J. Pooce

\section{NEUTRON THERMALIZATION}

\section{The Slowing Down and Thermalization of Neutrons}

By M. M. R. Williams. Pp. xv +582 . (Amsterdam: North-Holland Publishing Company, 1966.) 60 guilders; $120 s$.

During the past ten years there has been intensive experimental and theoretical work on the behaviour of neutrons in moderators, and most of it is still available only by reference to the original papers. It is therefore pleasing to see that this is beginning to be collected in book form, the more so because this is now by and large a well understood subject and a book written at present can be expected to retain its validity for some considerable time to come.

Dr. Williams's approach is theoretical, but the results of experiments are freely quoted where they holp the argument. Explanations are everywhere clear and lucid, and sufficient detail is included to enable the reader with limited knowledge to follow the argument, without being so elementary as to bore the expert. The book deals with the life history of neutrons in materials-particularly materials found in thermal reactors-from their birth to their eventual capture, and is divided into two parts, covering the slowing down region, where up-scatter of neutrons may be neglected, and the "pseudo equilibrium" region where neutrons may also gain energy from the moderator and where the details of the binding of the moderator atoms become important. It is, perhaps, slightly surprising to find the latter placed first for no obvious reason.

The first chapters give a fairly detailed account of slow neutron scattering in solids, liquids and gases, explaining the underlying theory, the various models it has been convenient to use to represent moderators and the experimental basis for the present knowledge of the scattering kernels used to calculate neutron spectra. From here the author proceeds to deal with spectra in infinite media, leading up from the early use of synthetic kernels to the present position based on experiment. This section includes a very welcome simplified treatment of Corngold's analysis of the asymptotic spectra in the epithermal region. Neutron spectra in finite media are very fully treated and this part includes most of the discussion of time dependent spectra and the time decay of the neutron density in a finite system (including the diffusion cooling phenomenon). Detailed analyses of the treatment of time and spatial dependence of spectra are included, but numerical methods of computing spectra are condensed into only three pages. Similarly, the chapter on actual reactor cell calculations and the sensitivity of cell parameters to the neutron spectrum seems short compared with the preceding sections, and even within this the use of computers is sometimes subordinated to analytical solutions.

Part II, dealing with spectra in the slowing down region, is almost two-thirds the length of Part $I$, which at first gives an unfair impression of the relative amount of work that has been devoted to spectra in this region. This possibly arises because this region is more easily handled analytically-reflecting the author's preference for the analytical approach-and also because a treatment of resonance capture and resonance escape probability in lattices is included. Nevertheless, the subject is thoroughly and clearly explained in a way that will make this book a valuable reference work.

The book is rounded off by a useful series of appendices dealing with a few specific topics in more detail and

\section{MOSSBAUER SPECTROSCOPY}

\section{Mössbauer Effect Methodology}

Vol. 1. Edited by Irwin J. Gruverman. (Proceedings of the First Symposium, New York City, January 26, 1965.) Pp. viii + 200. (Boston, Mass.: New England Nuclear Corporation, 1965. Distributed by Plenum Press, New York.) \$12.50.

THIs volume is a welcome addition to the growing number of books on various aspects of Mössbauer spectroscopy. It is the first to deal specifically with the experimental techniques of $\gamma$-ray resonance fluorescence spectroscopy and will be particularly helpful to the growing number of chemists, physicists and technologists who are becoming interested in this rapidly developing field. The editor and his colleagues are to be congratulated on devising such a balanced symposium and for publishing its proceedings so promptly. The fifteen papers have an average length of 12.5 pages and fall naturally into four main sections.

The first section consists of three reviews which are intended to indicate some of the current applications of the Mössbauer effect. R. H. Herber discusses recent applications to chemical problems; the title is somewhat misleading, since half the paper is concerned with the problems of standards, calibration and interconversion of data, but it provides a most useful summary of this important practical aspect of chemical Mössbauer spectroscopy. S. L. Ruby reviews the advances in physics and gives a brief but perceptive account of how developments have proceeded in magnetism, structure, lattice dynamics and nuclear physics. U. Gonser and R. W. Grant, in the longest article in the book (24 pages), give a comprehensive review of applications to biological systems.

The second section deals with the three main types of spectrometer and assesses the advantages and limitations of each. The articles also contain a wealth of useful practical information. E. Kankeleit discusses feedback in electromechanical drive systems and also deals with the problem of the accurate calibration of high-speed transducers. A. J. Bearden, M. G. Hauser and P. L. Mattern contribute a paper on their cam-driven, constant acceleration spectrometer, and P. Flinn puts in a gallant plea for the somewhat overlooked constant velocity drive systems.

Section 3 comprises five articles on the techniques of measurement. J. K. Major outlines the principles of measurement using scattering geometry and reviews previous results obtained by scattering techniques. This topic is developed further by P. Debrunner, who describes the scattering techniques used by the Illinois group; this work led to the detection of resonance scattering of the $155 \mathrm{keV} \gamma$-rays of osmium-188, the highest energy transition for which the Mössbauer effect has yet been observed. J. G. Dash reports techniques for measuring recoil-free fractions, $f$, by means of black absorbers, that is absorbers which are completely opaque to the Mössbauer line but perfectly transparent to all other radiation. Precision standardization of the differential chemical shift for iron-57 as developed at the National Bureau of Standards is reported by J. J. Spijkerman, F. C. Ruegg and J. R. De Voe. G. R. Gabriel discusses the computation of Mössbauer spectra: computer programmes are described which calculate the intensity and polarization of an emitted $\gamma$-ray or the absorption cross-section of the polarization is known.

The final section is devoted to means of controlling the environment: superconducting magnets ( $P$. P. Craig); 